\title{
How the COVID-19 pandemic has affected rheumatology research
}

\section{Paula Alba Moreyra, Francis Berenbaum (1), Debashish Danda, Bettina Grötsch, Simon R. Stones (1) and Sowmya Viswanathan (1)}

The COVID-19 pandemic has put pressure on researchers around the world. In this Viewpoint, six rheumatology researchers at different career stages and from different regions discuss the difficulty of conducting research during the pandemic, and also reflect on how the pandemic has changed their attitudes towards research and their plans for the future.

Q Since the COVID-19 pandemic began, what have been the biggest challenges to conducting your research?

Simon R. Stones. As a qualitative researcher in rheumatology and a patient living with rheumatic and musculoskeletal diseases, there have been several challenges to conducting research in my field. Prior to the UK Prime Minister's request to stop non-essential contact and travel in March 2020 , there was great confusion, and I was often conflicted between my researcher and patient roles. As a researcher, I was committed to my research participants and wanted to proceed as planned with data collection; however, my vulnerability as an immunocompromised patient caused great anxiety - both for my own wellbeing and for that of immunocompromised research participants.

Bettina Grötsch. When the German federal and state governments decided on measures to contain the spread of coronavirus in March 2020, I was still on parental leave with my second child and only came back to my normal work routine in June 2020 when the daily COVID-19 case numbers had declined. At that point, everyone was instructed to work from home whenever possible to stick to the contact restrictions. However, as I had to take care of my students in the lab, working from home was not a good solution for me. I really appreciated that our whole lab tried to continue our work at the bench-side in a normal way, but as it is quite hard to keep your distance in a small lab with many people around, a part of me was always afraid of becoming infected and especially of bringing this virus back home to my family. I have also had to deal with closed day care facilities and several quarantine isolations for both of my children that repeatedly forced me to coordinate my work from home with two small children around me.

Paula Alba Moreyra. The Argentine government had instituted a full lockdown by the end of March 2020 that affected the whole country when the first cases of COVID-19 were reported. My professional life and clinical practice has completely changed since then. During the first 3 months after the full lockdown, all routine appointments were cancelled and an emergency clinic was available twice a week. I work in an academic institution, not only running clinics but also doing clinical and basic research and educating both undergraduate and postgraduate students. The academic work was adapted to a web-based learning process and we continued with our clinical research with a lot of changes and difficulties.

Debashish Danda. Our research activities outside of COVID-19-related areas have been greatly affected in India, as patients with autoimmune and rheumatic diseases were unable to reach hospital unless they faced a rheumatological or COVID-19-related emergency. Therefore, our clinical and translational research were substantially curtailed owing to a shortage of clinical and biological materials. Another big challenge we faced was a drastic cut in research funding for non-COVID-19 areas as the bulk of existing funding was diverted to COVID-19-related research. As all of our younger staff were on COVID-19 duties and some research staff were unable to attend work owing to lockdowns and fear of exposure to COVID-19 in hospital, human resources were also compromised. In addition, all international and national visits related to collaborative research were and still are badly affected, which has been one of the biggest personal losses for many, including me.

Francis Berenbaum. The effects of the COVID-19 pandemic on the activity of my research laboratory have been phenomenal. We conduct experimental research that requires the handling of biological products and of small animals. Moreover, we use fresh joint tissues coming from patients at the time of joint replacement. As this type of surgery was withdrawn for many months, we had to stop doing this kind of research. The successive confinements that we experienced in France forced the majority of my team to stay at home. We managed to negotiate with our university the possibility for our $\mathrm{PhD}$ and post-doc students to come to the laboratory. To interrupt research for several months is to risk having to postpone one's professional project and sometimes also any associated private projects, so you can imagine the level of anxiety that these students had, given that their careers essentially depend on their publications.

Sowmya Viswanathan. As a scientist working in Canada on cellular and immune therapies for the treatment of osteoarthritis, being in a hospital setting (our institute is located within a hospital complex) has been a mixed blessing. Re-opening has been faster for us, but we have had stricter restrictions on spacing, shift work and capacity limits than our university-based counterparts. When we re-opened at $25 \%$ of normal capacity in June 2020 to 6-h shift work, it took an incredible amount of detailed planning and coordination between lab members for each experiment to be performed. My lab works on primary tissues 
and cells, and these certainly do not respect any shift-work boundaries. Funding has also been tight. Many of the new calls for proposals both from governments and from industry are rightly focused on COVID-19 research, but this means that fundamental and translational research on chronic diseases such as osteoarthritis has become a lower priority. Philanthropic funding, which can always be unreliable, has also been difficult to come by owing to cancelled fundraising events. Fortunately, government subsidies for trainee stipends have helped to cover some of the costs, particularly during the full shutdown.

Q How have your research activities changed? What adaptations have you put in place?

Simon R. Stones. During the initial period of the UK lockdown, the National Institute for Health Research's Clinical Research Network paused the set-up of new or ongoing studies that were not nationally prioritized COVID-19 studies. All parts of the research system were encouraged to help these COVID-19 studies to progress and to enable clinicians to be redeployed to support frontline care. Alongside the added pressures caused by the pandemic, such as home schooling, the most logical and sensitive action for my research was to stop recruitment of study participants and continue data collection online. Meetings initially stopped for several weeks as people adjusted to life in lockdown. My research activities used to involve frequent travel to other countries too, so that all stopped.

\section{Paula Alba Moreyra. Our research} activities have completely changed since the start of the COVID-19 pandemic. Telemedicine became a partial medical solution to respond to the pandemic in some aspects and to help clinical research. We had to introduce remote patient assessment, as well as local routine laboratory evaluation. Although it was very difficult to recruit new patients to trials and clinical and basic studies, we were able to continue with the patients we had already recruited. Some evaluations and disease activity assessments of our patients with rheumatoid arthritis or systemic lupus erythematosus were not done in the full lockdown and were instead scheduled for after lockdown was partially

\section{The contributors}

\section{Paula Alba Moreyra}

Paula Alba Moreyra is an Associate Professor of Rheumatology at Universidad Nacional de Córdoba and Director of the Rheumatology Unit at Córdoba Hospital in Córdoba, Argentina. Her clinical and translational research interests include systemic lupus erythematosus, antiphospholipid antibody syndrome, and pregnancy and autoimmune diseases.

\section{Francis Berenbaum}

Francis Berenbaum is a Professor of Rheumatology at Sorbonne Universite and Director of the Department of Rheumatology at AP-HP Saint-Antoine Hospital in Paris, France. He leads an experimental team at INSERM, and his research interests include understanding the relationships between metabolic diseases and osteoarthritis and new targeted therapies for treating symptoms and structural changes in osteoarthritis.

\section{Debashish Danda}

Debashish Danda is a Professor at and founder of the Department of Clinical Immunology \& Rheumatology at Christian Medical College \& Hospital in Vellore, India. He is the current President of APLAR. His clinical and translational research interests include Takayasu arteritis, Sjögren syndrome, spondyloarthritis and systemic lupus erythematosus.

\section{Bettina Grötsch}

Bettina Grötsch is a junior research group leader at Friedrich Alexander University ErlangenNuremberg in Erlangen, Germany. Her main research aim is to improve understanding of the complex interactions between the immune system and bone to help define new biomarkers and treatment strategies in autoimmune diseases such as rheumatoid arthritis.

\section{Simon R. Stones}

Simon R. Stones is a medical writer at Envision Pharma Group. He completed his PhD in applied health research and rheumatology at the University of Leeds in Leeds, UK. His main research interests are paediatric-onset inflammatory arthritis, self-management, digital health and patient engagement. He is also regarded as an international patient leader in rheumatology.

\section{Sowmya Viswanathan}

Sowmya Viswanathan is a scientist at the Krembil Research Institute and Associate Professor at the University of Toronto in Toronto, Ontario, Canada. Her research is focused on developing novel cellular and immune therapies to treat osteoarthritis and she is conducting Canada's first clinical trial using mesenchymal stromal cells for osteoarthritis.

lifted. In spite of the fact that we could not get the samples we needed for our clinical research, we were able to use telemedicine for other evaluations, such as health-related quality of life assessment. All research laboratories were closed during 2020 and all the samples from our collaborative research were stored and not sent for analysis by the core laboratory until the second COVID-19 wave was under control.

\section{Debashish Danda. The COVID-19}

pandemic has been and still continues to be the nightmare of our lifetime. The fear and panic kept most senior rheumatologists home-bound (especially those aged over 60 years) before vaccination was available, and many had to adopt an alternative plan to maintain their academic pursuits. Ongoing lab-based research with stored samples could be sped towards completion, if support staff were available. I decided to write up some of my pending manuscripts when the data were ready and strengthen my knowledge by reading COVID-19-related literature, particularly about its possible biological effects on autoimmune rheumatic diseases and other sequelae. Reviewing the existing literature and writing some narrative reviews was another strategy ${ }^{1}$. Questionnaire-based data generation with my existing cohorts of patients with autoimmune rheumatic diseases was another strategy, and I am currently trying to create some short communications and correspondences on the basis of that data. As a senior rheumatologist, I was also part of a couple of task forces and was involved in Delphi exercises and recommendations related to rheumatic diseases, anti-inflammatory treatment and vaccination-related issues, some of which have been published ${ }^{2}$.

Sowmya Viswanathan. As a team, we had to be nimble and responsive. The morning shift would often prime and harvest cells and tissues that would then be run in experimental set-ups by members of the afternoon shift; students would help each other on completely unrelated projects. This teamwork and collaboration was a happy by-product of the pandemic-imposed restrictions, and resulted in my lab members becoming more versatile and cross-trained in various techniques and concepts compared with when we worked on siloed projects. Another key crunch on resources was the availability of primary cells and tissues. We rely on healthy volunteers to donate blood and bone marrow, and on patients with arthritis for joint tissues. The second and even deadlier third waves in 
Canada meant that all elective surgeries were cancelled, and we had no access to patient samples. Normal hospital visits were non-existent, and research staff were only on site to conduct essential experiments. No one had the time or interest to consent to and donate tissue for research. We had to be creative and come up with alternative solutions, such as using stem cells to derive the primary cells needed for our in vitro models, re-framing research questions and shifting our focus, working with cell lines or jumping straight into animal models, or having to purchase primary cells from commercial vendors, which was an expensive option.

Bettina Grötsch. My research activities did not really change during the pandemic situation. Our lab was lucky that the pandemic did not affect our research. Hence, I continued my work in the field of osteoimmunology. Only at the beginning of 2021 did we face some delivery shortages for our consumables, especially those containing filters. This shortage has forced us to use our consumables sparingly and to organize our lab space, which is actually a positive development. In addition, like many labs, we have moved all our meetings and educational work to virtual work environments. Hence, research and education have become much less interactive and it has been a big challenge to accommodate this change. As a result of these virtual meetings, interaction between the students in the lab has been lost, and we are realizing that this is quite hard to re-establish.

Q Has the COVID-19 pandemic affected the quality of your research or your ability to publish? If so, what adaptations or allowances do you think are needed to account for the effects of the pandemic?

Simon R. Stones. I do not believe that the quality of my research has been affected by the pandemic, although I would attribute this to an inherent resilience in the face of adversity, which I share with many researchers forced to adapt to new and evolving situations. However, I do believe that the pandemic has influenced my findings. On the one hand, the pandemic has shed light on pertinent issues that might not have previously been discussed. On the other hand, I have had fewer opportunities to interact with research participants. As a qualitative researcher, building a rapport with participants is an essential part of the research process; ensuring that they feel comfortable sharing their experiences, while minimizing the researcher-participant power imbalance ${ }^{3}$. Although online methods can support these interactions, especially as more people become familiar with using the technology, it can be difficult to replace human interactions in a face-to-face setting, where non-verbal signs of communication can be interpreted. Although I have not personally observed a direct effect on publishing, I do think that the pandemic has revealed several flaws in the current peer-review process, which relies heavily on the generosity and capacity of already stretched clinicians and researchers although that is a topic for a separate discussion entirely!

Sowmya Viswanathan. Fundamentally, the pandemic restrictions have shifted how research is planned and conducted in my lab. I had always emphasized planning and mapping out all experiments, but these exercises have become even more critical now as we have had so little time to do experiments, and every hour in the lab had to count. Additionally, even as the lab capacity limits grew to $50 \%$ of normal, shortages of reagents became a major work-stoppage issue. My lab relies on PCR as a readout. Reagents such as RNA isolation kits, PCR master mixes, PCR plates and pipette tips, which would normally be available immediately or within $24 \mathrm{~h}$, were now at a 6-week minimum, or worse, indefinite, delay. This delay meant switching reagents mid-experiment and essentially repeating the first half to get uniform, interpretable results, or waiting out the delay to prevent re-doing half the dataset. The necessity of every experiment was called into question. How would the results from this experiment advance our learning? Did it support our fundamental hypotheses? Would it help us to fill in missing gaps for a manuscript in preparation? I had to be a ruthless tactician and cut down any 'let's see what happens'-type experiments (which, as most researchers know, generate serendipitously promising results), and only allow the most informative, urgent and useful experiments to proceed.

Bettina Grötsch. For me personally, the COVID-19 pandemic did not affect the quality of my research that much. I was even able to successfully apply for a research grant during the pandemic. However, in general, the turnaround time for research applications and for publications has slowed. Furthermore, our whole research group realized that it has become quite difficult to publish non-COVID-19-related research in high-quality journals during the past 2 years. Of course, research articles that help to understand or even show how to fight coronavirus are of great importance right now. However, from my point of view, we should not forget that there are still more diseases out there that urgently need basic and clinical research to improve the quality of life of those affected. Therefore, all journals need to adapt to the new situation and create space for both COVID-19 and non-COVID-19 research.

Francis Berenbaum. Thankfully, there has been no effect as such on our ability to publish, but we have had to be extremely reactive and orient our research forces towards a brand new theme - SARS-CoV-2 infection - as specific grants were allocated for this topic by our university. From osteoarthritis to COVID-19, it was not an obvious move!

Will the COVID-19 pandemic change your research activities for good, or will you return to business as usual once the situation is back to 'normal'?

Debashish Danda. Life will not be same again, especially for senior rheumatologists, as crucial time has been lost. Once the situation changes back to normal, we can try to be back to business as usual (in terms of research), if opportunities are provided. However, some of the modalities of research activities that were adopted during the COVID-19 pandemic can be continued too, as these adaptations have indeed been proven to be newly discovered opportunities during these challenging times. For example, real-world research that makes use of digital technologies, telemedicine or databases might become an ongoing activity in the future. COVID-19-related clinical research might also continue in many fields, including rheumatology, for the next few years, even after the pandemic ends $s^{4,5}$. Interesting ongoing research questions include COVID-19 as a trigger for the onset of autoimmunity, as well as how exposure to COVID-19 and COVID-19 vaccinations will change the outcome of autoimmune rheumatic diseases.

Paula Alba Moreyra. Now that advances have been made in the vaccination programme, we are returning to usual research and clinical activities while maintaining all protective measures. Technological innovations have become an important resource for the care of 
patients with rheumatic diseases, for continuing medical education online and for maintaining research networks during the pandemic. However, in spite of the important role of telemedicine during the pandemic, we welcome the return to face-to-face appointments, particularly for those patients with severe or difficult to treat disease. Although the time we have been able to devote to research activities has not been enough, new research opportunities have opened up, such as the study of the prevalence, clinical manifestations and outcomes of COVID-19 infection in patients with rheumatic diseases. The Argentine Society of Rheumatology has developed two registries, the first to learn the effect of SARS-CoV-2 on patients with rheumatic diseases in Argentina, and the second to evaluate the safety and efficacy of COVID-19 vaccines in this population. Our rheumatology unit has actively participated in these two projects and in other projects at the Universidad Nacional de Córdoba.

Sowmya Viswanathan. For my lab, the pandemic has changed our approach to research. It has increased, not diminished, the quality of research, as we have had to be razor-focused on our research questions and have been careful to design every run of experiments with appropriate controls to generate useable and interpretable data. We have trimmed all excesses. The restrictions imposed by shift work, limited reagents and the availability of cells and tissues have also made us re-evaluate experimental approaches and re-examine and question hypotheses, as well as foster more critical thinking. This mind-set is now being passed on to new trainees joining the lab, and even though shift-work restrictions have eased, we are very mindful that small changes in how the pandemic is managed could reverse all gains. Our experiments will continue to be planned and executed around rationed resources and, ultimately, I think that this makes us better researchers.

Francis Berenbaum. Not everything has been negative: collaborations have been set up with teams we would never have imagined working with before the pandemic. For example, our expertise on the biology of prostaglandins led us to collaborate with a team that was working on the antiviral properties of an NSAID ${ }^{6}$. Similarly, our expertise on the cholinergic system led to a joint publication with researchers from the Pasteur Institute and the Cochin Hospital on the role of this system in the hyperinflammatory state of patients with COVID-19 (REF.7). Not to mention the wonderful international exchanges I have had within the framework of the COVID-19 Global Rheumatology Alliance registry ${ }^{8}$. The pandemic has also expanded our communication capabilities. Thanks to improvements in the quality of videoconferencing platforms, we realized that certain small working meetings between disseminated research teams could be held remotely without any loss of quality and with considerable time savings as travel was avoided. However, this pandemic has also shown us that virtual large meetings will never replace the usual face-to-face meetings. Indeed, even if virtual meetings allow us to present our work to the rest of our research community, the lack of user-friendliness and interactivity greatly reduces their interest. Research is also done around the coffee machine!

Simon R. Stones. I do not think that we will ever get back to normal, only to a 'new normal' that we all must learn to embrace. I believe that COVID-19 has made many individuals reassess their work-life balance. Pre-pandemic, I was travelling excessively for various meetings. As much as I despised the national lockdowns that were imposed in 2020 , those periods of confinement enabled me to reflect on my life and its fragility, as I witnessed so many people prematurely lose their lives. I also believe that this forced change has come at a time when we must collectively take greater action to tackle pressing issues such as climate change. Admittedly, some of the meetings I would travel abroad for can quite easily be conducted online. Not only is this better for the environment, but it also saves me a considerable amount of time and effort and can be more economical too. That is not to say that all meetings should become virtual, but I do think that greater attention is required during the planning process. In terms of how research is conducted, I believe that the pandemic has accelerated the use of innovative methods in research, many of which were once described as too complex, showing that anything is possible. I also believe that greater flexibility and choice are just two reasons why a more open and dynamic approach to research could be beneficial for everyone involved.

Q Looking to the future, how do you think that the COVID-19 pandemic will affect your career? Has it made you consider alternative career paths or created unexpected opportunities?
Bettina Grötsch. The COVID-19 pandemic has indeed slowed down my career plans owing to a combination of the restrictions in the lab, the long processing times for applications and publications, as well as taking care of my children during the lockdown in the winter months. However, I am still a young researcher and have only just started my independent academic career. Therefore, I am really looking forward to a future without COVID-19 restrictions, not only for pushing my career forward but also for improving the work-life balance for all families.

Debashish Danda. I am not sure if the COVID-19 pandemic has created any alternative career paths for me; if anything, it has made my current path more difficult. I was looking forward to several international collaborations, research sabbatical opportunities and exchange visits to strengthen my research. At over 60 years of age and with 2 years lost, my academic career has been badly affected, particularly by the loss of assistants. Government and private research funding to very senior researchers is highly restricted, if not non-existent, in India. This is a painful fact that is often not given any consideration by many institutes, funding bodies and even colleagues. One of the most crucial periods of my career has been washed away by the COVID-19 pandemic; therefore, I think that career paths need to be assessed from a pre-COVID-19 time-point, rather than being counted chronologically. I am looking forward to a career now where I can build new academic, teaching and training facilities with some lead research roles, and to opportunities to catch up the time lost by upskilling myself appropriately for this new scenario. Societal leadership and social research related to my speciality is another area where I wish to contribute, if opportunities arise.

Simon R. Stones. During the COVID-19 pandemic, I have defended my PhD thesis and transitioned from academia to the pharmaceutical industry as a medical writer. I feel blessed to be able to work remotely from home in a role that offers flexibility, although I know that this is not the case for many. The pandemic provided countless opportunities for me to reflect and consider how I could best use my skills and experiences to have the greatest possible influence, which guided me into medical communications. Different experiences throughout the pandemic have shown how much people rely on different media to digest health-related information, and so 
I now see it as part of my duty to help craft and disseminate accurate information in an accessible format for everyone, regardless of their background.

Francis Berenbaum. Being a professor with a tenured position, this pandemic will not affect my career as such. But it has definitely created new interactions with teams outside my usual field of research. The future is indeed interdisciplinary!

Paula Alba Moreyra ${ }^{1,2}$, Francis Berenbaum (iD ${ }^{3 凶}$, Debashish Danda ${ }^{4 \times}$, Bettina Grötsch ${ }^{5 凶}$, Simon R. Stones (iD ${ }^{6,7 凶}$ and Sowmya Viswanathan (D) $^{8,9 凶}$ ${ }^{1}$ Rheumatology Unit, Córdoba Hospital, Córdoba, Argentina.

${ }^{2}$ Facultad de Medicina, Universidad Nacional de Córdoba, Córdoba, Argentina.

${ }^{3}$ Sorbonne University, INSERM, AP-HP Saint-Antoine Hospital, Paris, France.

${ }^{4}$ Department of Clinical Immunology \& Rheumatology, Christian Medical College \& Hospital, Vellore, Tamil Nadu, India.

${ }^{5}$ Department of Medicine 3, Rheumatology and Immunology, Friedrich Alexander University
Erlangen-Nuremberg and Universitätsklinikum Erlangen, Erlangen, Germany.

${ }^{6}$ Engage Scientific Solutions, Envision Pharma Group, Wilmslow, UK

${ }^{7}$ School of Healthcare, University of Leeds, Leeds, UK.

${ }^{8}$ Krembil Research Institute, University Health Network, Toronto, Ontario, Canada

9 Institute of Biomedical Engineering

Division of Hematology, Department of Medicine University of Toronto, Toronto, Ontario, Canada.

凶e-mail:paulaalba@yahoo.com; francis.berenbaum@ aphp.fr; debashisdandacmc@hotmail.com; bettina.groetsch@uk-erlangen.de;simon@ simonstones.com; sowmya.viswanathan@ uhnresearch.ca

https://doi.org/10.1038/s41584-021-00735-7

Published online 11 January 2022

1. Shah, S. et al. Autoimmune and rheumatic musculoskeletal diseases as a consequence of SARS-CoV- 2 infection and its treatment. Rheumatol. Int. 40, 1539-1554 (2020).

2. Tam, L.-S. et al. Updated APLAR consensus statements on care for patients with rheumatic diseases during the COVID-19 pandemic. Int. J. Rheum. Dis. 24, 733-745 (2021).

3. Tremblay, S. et al. Conducting qualitative research to respond to COVID-19 challenges: reflections for the present and beyond. Int. J. Qual. Methods 20, 1-8 https://doi.org/10.1177/16094069211009679 (2021).
4. Yazdany, J. COVID-19 in rheumatic diseases: a research agenda Arthritis Rheumatol. 72 1596-1599 (2020).

5. Hyrich, K. L. \& Machado, P. M. Rheumatic disease and COVID-19: epidemiology and outcomes. Nat. Rev. Rheumatol. 17, 71-72 (2021).

6. Terrier, O. et al. Antiviral properties of the NSAID drug naproxen targeting the nucleoprotein of SARS-CoV-2 coronavirus. Molecules 26, 2593 (2021).

7. Courties, A. et al. Regulation of the acetylcholine/ a $7 n A C h R$ anti-inflammatory pathway in COVID-19 patients. Sci. Rep. 11, 11886 (2021).

8. Kim, A. H. J. et al. A rush to judgment? Rapid reporting and dissemination of results and its consequences regarding the use of hydroxychloroquine for COVID-19. Ann. Intern. Med. 172, 819-821 (2020).

\section{Acknowledgements}

B.G. thanks the whole AG Bozec group and especially A. Bozec, who always supported her during her career.

\section{Competing interests}

S.R.S. is an employee of Envision Pharma Group, owns stock options in Envision Pharma Group, has served as a consultant for 67 Health, Ampersand Health, Envision Pharma Group, Janssen, On The Pulse Consultancy, Parexel, Sheffield Hallam University and the University of Aberdeen, and is a trustee of RAilSE, a charitable incorporated organization registered in England and Wales. The other authors declare no competing interests

Publisher's note

Springer Nature remains neutral with regard to jurisdictional claims in published maps and institutional affiliations.

(C) Springer Nature Limited 2022 